\title{
Análisis Técnico, Económico y Medioambiental de la Fabricación de Bloques de Hormigón con Polietileno Tereftalato Reciclado (PET)
}

\author{
Josefina Infante-Alcalde y Claudia Valderrama-Ulloa* \\ Escuela de Construcción Civil, Facultad de Ingeniería, Pontificia Universidad Católica de Chile, Av. Vicuña Mackenna \\ 4860, Macul, Santiago - Chile. (e-mail: jinfant4@uc.cl, c.valderrama@uc.cl)
}

* Autor a quien debe ser dirigida la correspondencia

Recibido Ene. 15, 2019; Aceptado Mar. 13, 2019; Versión final Abr. 5, 2019, Publicado Oct. 2019

\section{Resumen}

Se propone la reutilización de material plástico en reemplazo del agregado fino en la fabricación de bloques de hormigón. Se analiza el comportamiento técnico, económico y medioambiental de la propuesta, a través de ensayos mecánicos, comparación de precios del mercado y cálculo de huella carbono. Técnicamente se encontró que las probetas de hormigón con un $10 \%$ de reemplazo PET tienen propiedades mecánicas acorde con la normativa chilena para bloques no estructurales, lo que permitiría la construcción de muros no estructurales. El análisis económico muestra que este bloque es altamente competitivo en comparación a los productos presentes en el mercado chileno. Finalmente, el cálculo de la huella carbono en las diferentes etapas de su ciclo de vida presenta una disminución en la etapa de extracción de materias primas.

Palabras clave: bloques; residuos; plásticos; ciclo de vida; costos de construcción; huella del carbono

\section{Technical, Economic and Environmental Analysis of the manufacture of concrete blocks with Recycled Terephthalate Polyethylene (PET)}

\begin{abstract}
It is proposed the reuse of plastic in concrete blocks in replacement of fine aggregates. The technical, economic and environmental behavior of the proposal is analyzed, through mechanical tests, comparison of market prices and calculation of carbon footprint. Technically it was found that concrete specimens with $10 \%$ PET replacement have mechanical properties in accordance with Chilean regulation for no-structural blocks, which would allow the construction on non-structural walls. Economic analysis shows that concrete blocks is highly competitive compared to the products already on the Chilean market. Finally, the carbon footprint calculation on life cycle shows a decrease in the extraction stage of raw materials.
\end{abstract}

Keywords: blocks; waste; plastic; life cycle; construction costs; carbon footprint 


\section{INTRODUCCIÓN}

La industria de la construcción es uno de los sectores que más contamina, es por ello que debe ser más consciente con el medioambiente creando tecnologías eficientes y ecológicas que minimicen los impactos medioambientales y sean sustentables en el tiempo, así como también buscar la opción de aprovecharlos para una segunda vida.En el sector de construcción chileno uno de los materiales más utilizados es el hormigón, debido a sus excelentes características físicas, como durabilidad, resistencia a la compresión y trabajabilidad del material en estado fresco. No obstante, su uso también acarrea unos grandes costes medioambientales, por la enorme cantidad de energía consumida y $\mathrm{CO}_{2}$ liberado durante su fabricación. Además, la obtención de áridos necesarias para la obtención del cemento, puede implicar la destrucción de ciertos hábitats. Por otra lado los residuos también generan un gran impacto al medio ambiente, sobre todo aquellos de lenta degradación como es el caso de los plásticos, los cuales se acumulan en vertederos, basurales o rellenos sanitarios, que con el tiempo forman parte de los lixiviados creando un peligro al contaminar aguas subterráneas. El consumo de plástico es cada vez mayor, Chile proyecta al 2020 una producción de envases plásticos de más de 860 mil toneladas (C y V Medioambiente, 2010) y las cifras de que sólo un $4 \%$ está siendo reciclado son realmente alarmante.

Es así que consciente de los problemas medioambientales que acarrean los desechos industriales en el sector construcción, numerosas son las investigaciones que han buscado agregar estos residuos al hormigón para mejorar algunas de sus propiedades (Pacheco-Torgal et al., 2012, como fibras poliméricas (Vélez-Herrera et al., 2016) o naturales (Martínez-Barrera et al., 2013 o Guo et al., 2018), polipropileno (Yang et al., 2015), Nylon (Orasutthikul et al., 2017), caucho de neumáticos (Gupta et al., 2014), residuos plásticos de envoltorios y adiciones de Acrilonitrilo Butadieno Estireno ABS (García de los Santos et al., 2013), adiciones de Policloruro de Vinilo PVC (Costa del Pozo, 2012). Y también están los estudios del análisis de la factibilidad de fabricar hormigones más livianos con residuos de Polietileno Tereftalato PET para mejorar la aislación térmica (Gaggino et al., 2007) o mejorar su porosidad (Mendivil-Escalante, 2017), para mejorar y evaluar al ataque de sulfuros (Nikbin et al., 2016), con bolsas de plásticos para mejorar la permeabilidad a la penetración de cloruros, la carbonatación y la absorción capilar (Gómez et al., 2011), PET provenientes de botellas recicladas y que se comparan con fibras de polipropileno (Kim et al., 2009) o PET que se comparan con polietileno de alta densidad (Tafheem et al., 2018).

Finalmente en la investigación Choi et al. (2005) se estudiaron los efectos de tereftalato de polietileno (PET) en la densidad del hormigón. Se planificaron las proporciones de mezcla de hormigón de modo que las relaciones agua-cemento fueron $45 \%, 49 \%$ y $53 \%$, y las tasas de sustitución de WPLA eran $0 \%, 25 \%, 50 \%$ y $75 \%$ en volumen de agregado fino. La densidad de las mezclas de concreto disminuyó con el aumento en el contenido WPLA y en la investigación de Saikia y de Brito (2014) se comprueba que la incorporación de PET en el hormigón evita las fallas a someterlo a tracción y flexión al igual que mejora su comportamiento a la resistencia a la abrasión y finalmente en la investigación de (Alesmar et al., 2008), se utilizaron porcentajes de $5 \%, 10 \%$ y $15 \%$ de PET para mezclas de mortero y concreto. Se ensayaron probetas similares a las que se describirán en la investigación y que nos ayudarán a ratificar los resultados que se pretenden obtener aquí en cuanto a resistencias y absorción de agua.

Es así como el presente estudio está orientado a evaluar el efecto de agregar Polietileno Tereftalato (PET), proveniente del reciclaje de las botellas plásticas en el hormigón como aditivo en reemplazo a la arena y analizar sus consecuencias, tanto técnicas en la resistencia a compresión y a flexión y en la penetración de cloruros, pero además, a diferencia de las investigaciones ya citadas, se incorpora un análisis económico de la nueva solución frente a sus competidores más cercanos y así conocer su viabilidad y competencia en el mercado. Para finalmente abordar un análisis del aspecto medioambiental, analizado a través de la huella de carbono.

\section{MARCO TEÓRICO}

Chile lidera en Latinoamérica como el país que genera mayores cantidades de residuos, reciclándose solamente el 10\%, donde una gran mayoría es reciclaje de material ferroso (CONAMA, 2010). En el estudio de Comisión Nacional del Medio Ambiente (2010), se indica que para el año 2009 se generaron 16,9 millones de toneladas de residuos, de los cuales 6,5 millones de toneladas corresponden a residuos municipales, 4,58 millones de toneladas a residuos industriales y 5,82 millones de toneladas de residuos de construcción. De estos residuos es posible apreciar en la tabla 1, el gran potencial de reciclado que poseen, destacándose los áridos, cementos y ladrillos. También se debe destacar que en Chile se ha avanzado en la preocupación del destino final de los residuos, en 1995 la totalidad de los residuos domiciliarios se disponía en vertederos y basurales; en cambio al año 2005 , más del $60 \%$ de los residuos se disponen en rellenos sanitarios. Y al año 2009 un $69 \%$ se depositó en rellenos sanitarios, $22 \%$ en vertederos y $9 \%$ en basurales. 
Tabla 1:. Composición de los residuos generados de las actividades de construcción en Chile

\begin{tabular}{|l|c|l|c|}
\hline Residuos & Miles de toneladas & Residuos & Miles de toneladas \\
\hline Áridos & 4.599 & Cerámica muro & 4 \\
\hline Cementos & 653 & Pizarreño & 4 \\
\hline Ladrillos & 313 & Baldosa & 4 \\
\hline Madera & 28 & Tubos de acero & 2 \\
\hline Yeso & 24 & Zinc & 1 \\
\hline Fierro redondo & 20 & Azulejos & 1 \\
\hline Cañerías de cobre & 13 & Tubos de PVC & 142 \\
\hline Parquet & 9 & Otros & 2 \\
\hline
\end{tabular}

Para el $10 \%$ de residuos industriales y municipales que se logran reciclar en Chile, en la tabla 2 se observa su distribución en cuanto a tipo de residuos, su porcentaje valorizado al año 2009 y las toneladas generadas. Se destaca el alto porcentaje de metales valorizados, esto se debe a que el acero no pierde calidad al reciclarse, dando un alto potencial comercial a las empresas que reciclan metales. Y también se puede obserar los bajos índices de reciclaje de plástico en Chile, siendo que es una de los residuos más generados con una posible reutilización. Específicamente en el reciclaje de PET actualmente en Chile se está reciclando solo un $4 \%$ de los 668 mil toneladas, ocupando casi el último lugar de la valorización nacional y por muy debajo del $82 \%$ que presentan los materiales ferrosos. De lo anterior es que en la investigación se estudiará el impacto que tiene este material en el medio y sus posibilidades de reciclaje.

Tabla 2: Generación y porcentaje de valorización por tipo de residuo industrial y municipal

\begin{tabular}{|l|c|c|}
\hline Tipo de residuo & Generación (miles de tonS) & Valorización (\%) \\
\hline Material ferroso & 286 & $82 \%$ \\
\hline Papeles y cartones & 913 & $41 \%$ \\
\hline Alumnio & 10 & $40 \%$ \\
\hline Vidrio & 266 & $29 \%$ \\
\hline Plásticos & 668 & $4 \%$ \\
\hline Otros metales no ferrosos & 64 & $3 \%$ \\
\hline Tetraplak & 18 & $2 \%$ \\
\hline
\end{tabular}

\section{METODOLOGÍA}

A continuación se describen los diferentes métodos experimentales para analizar el comportamiento técnico de los bloques, luego se detallan los supuestos y alcances utilizados para analizar los costos asociados a la producción del elemento y finalmente se describen los pasos y consideraciones realizados para determinar el impacto medioambiental de los bloques a través del cálculo de emisiones de $\mathrm{CO}_{2}$ y energía utilizada en su ciclo de vida.

\section{Material y equipo experimental para el análisis técnico}

Las propiedades de la arena, gravilla y PET que se realizaron (a partir de las Normas Chilenas NCh1223, NCh1116, NCh1239, NCh1117 y NCh1326) fueron: \% de finos Densidad Aparente Compactada $\left(\mathrm{kg} / \mathrm{m}^{3}\right)$, Densidad Aparente Suelta $\left(\mathrm{kg} / \mathrm{m}^{3}\right)$, Densidad Real del árido saturado superficialmente seco $\left(\mathrm{kg} / \mathrm{m}^{3}\right)$, Densidad Real del árido seco $\left(\mathrm{kg} / \mathrm{m}^{3}\right)$, Densidad Neta $\left(\mathrm{kg} / \mathrm{m}^{3}\right)$, Absorción de Agua (\%) y Porcentaje de huecos (\%). Además, se determinaron las granulometrías de cada agregado según NCh165. Y el PET previamente triturado, utilizándose exclusivamente PET menor a $5 \mathrm{~mm}$. Para la confección de las probetas se utilizaron moldes metálicos prismáticos, de dimensiones $40 \times 40 \times 160 \mathrm{~mm}$. En total se confeccionaron 60 probetas Rilem, fabricadas 12 de cada dosificación (hormigón patrón, 5\%, 10\%, 15\% y 20\% de contenido PET en reemplazo de arena). Con el estudio de las granulometrías de los áridos y del PET, se determina reemplazar un porcentaje de arena por el PET que pasa bajo el tamiz de $5 \mathrm{~mm}$. Posteriormente las probetas son trasladadas a una cámara húmeda, sumergidas en agua detenida, saturada con cal, a $23 \pm 2{ }^{\circ} \mathrm{C}$ durante 28 días, hasta el momento de los ensayos. 
Para completar el análisis técnico se realizaron los siguientes ensayos de laboratorio: 1) Densidad, luego de ser curadas en sumersión por 28 días; 2) Ensayos a flexión y compresión de 3 probetas de cada muestra según la NCh158. Los ensayos son realizados a los 28 días de curado, se secan superficialmente y se procede a ensayar primero a flexión y luego con cada una de las partes de la probeta quebrada por la mitad se realizan los ensayos a compresión; 3) Porcentaje de absorción de agua: posterior al curado de las probetas éstas son secadas por $24 \mathrm{~h}$ en horno a una temperatura de $50 \pm 5^{\circ} \mathrm{C}$, luego de retiradas del horno éstas son pesadas hasta obtener masa constante y determinar su porcentaje; y finalmente 4) Penetración acelerada de cloruros (a 48, 96 y 144h, aquí solo se muestran los resultados de las 48h), siguiendo la norma sueca de Fagerlund de absorción capilar. Las probetas luego de su proceso de curado son secadas durante 24 horas en un horno a $50 \pm 5^{\circ} \mathrm{C}$, y se sumergen en una solución de agua con Cloruro de Sodio a una concentración de $35 \mathrm{~g} / \mathrm{l}$. Esta solución busca simular la concentración de sales de las aguas marinas en las costas chilenas.

\section{Supuestos y alcances para el análisis económico}

El reciclaje de plástico para ser utilizado como materia prima en la fabricación de materiales de la construcción trae consigo una incidencia económica en varios aspectos. Primero se está utilizando un desecho, por lo que es muy económico o bien no hay que pagar por ello, ya que se prescinde de su extracción y fabricación porque simplemente se está recuperando dicho material. De todos modos tiene costo en su recolección, clasificación y triturado o bien de la compra directamente a las empresas recicladoras del PET triturado, en este último caso tiende a ser más caro que el material que se pretende reemplazar, debido a los gastos operacionales y ganancias de la empresa, ya que actualmente las cantidades de reciclaje plástico no son lo suficientemente altas para fomentar economías de escala.

Para su fabricación no requiere de gran infraestructura ya que dependerá de la escala en que se quiera fabricar y no necesita de gran implemento, incluso se puede fabricar de manera artesanal. $O$ bien al tratarse de un sistema con posibilidades de industrializarse, este abarata costos de mano de obra y produce un sistema más eficiente. Se abaratan los costos de construcción ya que no requiere de mano de obra calificada para su implementación por basarse en ladrillos o bloques estos serán instalados como albañilería, incluso puede ser autoconstruido por las familias que vivirán en ella. Para el estudio económico se realizaron varios supuestos: 1) El precio de PET es para compras mayores a una tonelada y el material viene limpio y triturado para ser utilizado y 2) Los áridos incluyen el transporte al pie de obra.

\section{Consideraciones para el análisis medioambiental}

Se sabe que el Análisis de Ciclo de Vida (ACV) es una herramienta de gestión medioambiental que analiza todos los procesos de un producto o actividad, conociendo los recursos consumidos y los residuos que se generan, para así cuantificar las consecuencias medioambientales que generan las emisiones ligadas al producto. Pero el uso de esta herramienta trae consigo una serie de limitaciones: 1) algunas hipótesis o supuestos pueden ser subjetivos, ello por no conocer el comportamiento en el futuro que tiene el sistema; 2) los indicadores varían dependiendo de la región donde se encuentre, por lo que es muy difícil aplicar datos globales para aplicaciones locales; y 3) la precisión de los estudios se encuentra limitada por la accesibilidad o disponibilidad de ciertos datos. Es por estas razones que los ACV no se comparan entre productos, simplemente es para analizar el proceso independiente con el fin de racionalizar los recursos, reducir los consumos y disminuir los impactos ambientales.Es así como el objetivo y alcance de este análisis, es la identificación de las entradas energéticas y aspectos ambientales en la etapa de fabricación del producto, y además comparar el consumo energético de esta etapa con otras tales como la obtención de materias primas y la de operación o uso, de manera de clarificar la importancia del ACV en el producto.

La metodología utilizada obedece al principio normativo ISO 14040:2006. Para este estudio se enfocará el ACV desde las etapas de extracción de las materias primas hasta su fabricación, debido a que se trata de un material de construcción se puede continuar su estudio considerando transporte a la faena, construcción y posterior demolición, enfocado en calcular la huella de carbono a través del consumo energético en $\mathrm{kW} / \mathrm{h}$, considerando el consumo eléctrico utilizado en cada actividad y el consumo de las energías fósiles como petróleo y bencina, convirtiéndose este último dato en consumo eléctrico en cuanto al rendimiento de la máquinaria o equipo utilizado. La fabricación de ladrillos de hormigón con PET reciclado comienza su ciclo con la extracción y procesamiento de las materias primas, arena, gravilla y cemento. En el caso del PET al ser un material ya reciclado se prescinde de su fabricación. Luego todos los materiales son transportados al lugar de construcción, donde se confeccionarán los ladrillos a pie de obra. El ciclo continúa con la construcción de la vivienda, su uso y mantenimiento de 50 años (bajo el supuesto de que no existirá ningún sismo de gravedad) y su posterior demolición. Para el cálculo de la huella de carbono la gran mayoría de los datos fueron obtenidos bajo supuestos de documentos extranjeros, debido a la poca información que se tiene en Chile sobre los factores o conversiones a utilizar (Athena Sustainable Materials Institute, 1998) 


\section{RESULTADOS}

A continuación se presentan los diferentes análisis y resultados de la investigación: 1) en los resultados del aspecto técnico se discuten los valores obtenidos para la densidad, flexión, compresión, absorción de agua y penetración de cloruros; 2) en los resultados económicos se analiza los costos de fabricación y un comparativo con los principales competidores del productos; y 3) finalmente en los resultados medioambientales se analizan y discuten la cantidades de emisiones de $\mathrm{CO}_{2}$ equivalente, junto con el consumo energético para todo el ciclo de vida del producto, así como la incidencia de cada una de las materias primas en el proceso

\section{Resultados técnicos}

En la tabla 3 se pueden observar los diferentes resultados de los diferentes ensayos realizados:

\section{Densidad}

En relación a la densidad se puede apreciar una disminución constante a medida que aumenta el porcentaje de PET, pero para la probeta con $20 \%$ PET la variación de pérdida es mayor $(1,84 \%$ respecto a la probeta patrón). Los resultados obtenidos reflejan la literatura, ya que al agregar más PET en reemplazo de la arena la densidad disminuye por la densidad de este agregado (Gaggino et al., 2007), estos resultados reflejan un material un poco más ligero, más fácil de trabajar y que contribuya a un menor peso propio en la estructura.

Tabla 3: Resumen Resultados Ensayos Técnicos

\begin{tabular}{|l|c|r|r|r|c|}
\hline Ensayos & Patrón & $\begin{array}{c}\text { Mezcla } \\
5 \% \text { PET }\end{array}$ & $\begin{array}{c}\text { Mezcla } \\
10 \% \text { PET }\end{array}$ & $\begin{array}{c}\text { Mezcla } \\
15 \% \text { PET }\end{array}$ & $\begin{array}{c}\text { Mezcla } \\
20 \% \text { PET }\end{array}$ \\
\hline Densidad [kg/m³] & 2,21 & 2,21 & 2,21 & 2,20 & 2,17 \\
\hline Flexión [MPa] & 4,83 & 4,00 & 4,13 & 3,73 & 3,60 \\
\hline Compresión [MPa] & 26,1 & 23,4 & 24,1 & 21,4 & 21,7 \\
\hline Absorción [\%] & 7,81 & 7,61 & 7,26 & 7,11 & 7,52 \\
\hline Disminución porcentual [\%] & 0,00 & 2,65 & 7,05 & 8,93 & 3,79 \\
\hline Penetración Cloruros [\%] & 4,14 & 4,08 & 3,49 & 3,96 & 3,76 \\
\hline
\end{tabular}

\section{Ensayo flexión}

Los resultados para la resistencia a la flexión son muy constantes, todas las probetas con adición de PET presentan una disminución de resistencia, en promedio de un $20 \%$, la máxima variación de resistencia se presenta para la probeta de $20 \%$ PET con una variación de resistencia del $25 \%$ respecto a la mezcla patrón (Figura $\mathrm{N}^{\circ} 1$ ). Esto se puede deber a que esta cantidad de PET no permite la homogeneidad en la mezcla y no se produce el efecto en que las fibras sirven de puente para traspasar las cargas de un lado al otro de la grieta que se ha formado. La probeta con $10 \%$ PET mostró una buena respuesta respecto a la resistencia a flexión entre sus pares, donde disminuyó su resistencia de sólo un $14 \%$ respecto a la probeta patrón. Puede ser que la dosis en esta probeta sea la precisa para resistir a la flexión tal como se observó en el trabajo de Gómez et al., (2011). Se puede concluir que la fractura no se genera por adherencia entre la pasta de cemento y el PET, los trozos de plástico no se desprendían con facilidad y el área de corte es transversal a la probeta sin generarse desviaciones donde se encontraba el PET.

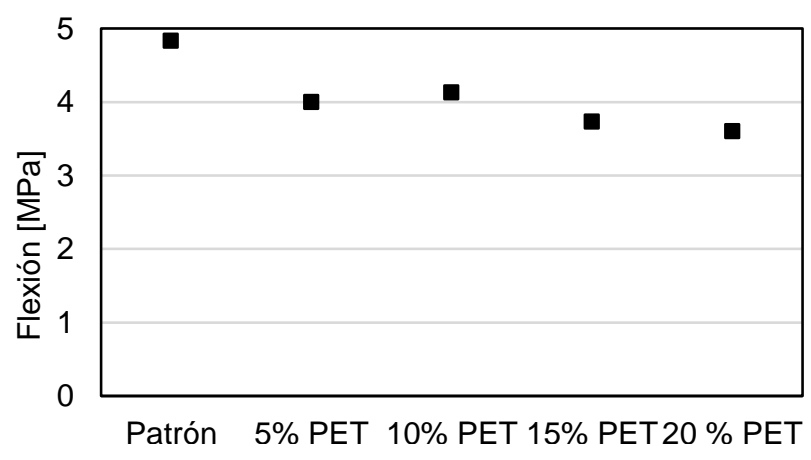

Fig. 1: Resultados ensayos de Flexión 
Las probetas se rompieron en su totalidad en el plano de falla (ver figura $\mathrm{N}^{\circ} 2$ ), de esto se extrae que los trozos de PET no transmiten la carga como ocurre con otras fibras de polímeros que sirven como puente para transmitir la carga de un lado de la grieta a otro para evitar su apertura. Esto se debe al tamaño y forma del polímero, ya que el largo es insuficiente para haber transmitido la carga, pero debido al espesor del PET esta solución es poco factible ya que no podría haberse homogenizado con la mezcla debido a que al aumentar la superficie específica de contacto con la pasta de cemento se habría generado segregación en la mezcla afectando sus propiedades físicas.

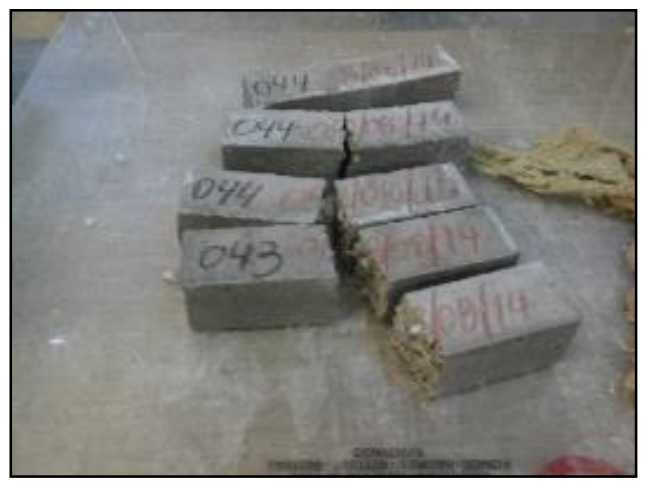

Fig. 2: Plano de Falla en Flexión

\section{Ensayo de compresión}

Sobre este ensayo se puede observar (figura $N^{\circ} 3$ ) que en todas las probetas con agregado PET se obtiene una baja en la resistencia a la compresión en relación a la probeta patrón, con una disminución en promedio de un $13 \%$, esto es debido a la flexibilidad del material, pues permite mayores deformaciones, debido al bajo módulo de elasticidad que posee y es de esperar que decrezca la fuera necesaria para deformar el material a medida que aumenta la adición de PET. Para la probeta con $10 \%$ se repite lo mismo que en la resistencia a la flexión, donde su variación porcentual respecto a la probeta patrón es la menor con un decrecimiento de $8 \%$, lo que podría explicarse en que la cantidad de PET se ha agregado en la correcta proporción y esto permite mantener una cierta uniformidad en la probeta y por tanto las fisuras formadas durante las etapas iniciales de carga son unidas por los trozos de PET. Estos resultados son satisfactorios ya que con la misma dosis de PET este responde positivamente a los ensayos de flexión y compresión. La falla producida no genera quiebre en ninguna de las probetas. Tal como se dijo anteriormente se puede reafirmar que la adición de PET no colabora a un desprendimiento con la pasta de cemento que facilite la disgregación del material.

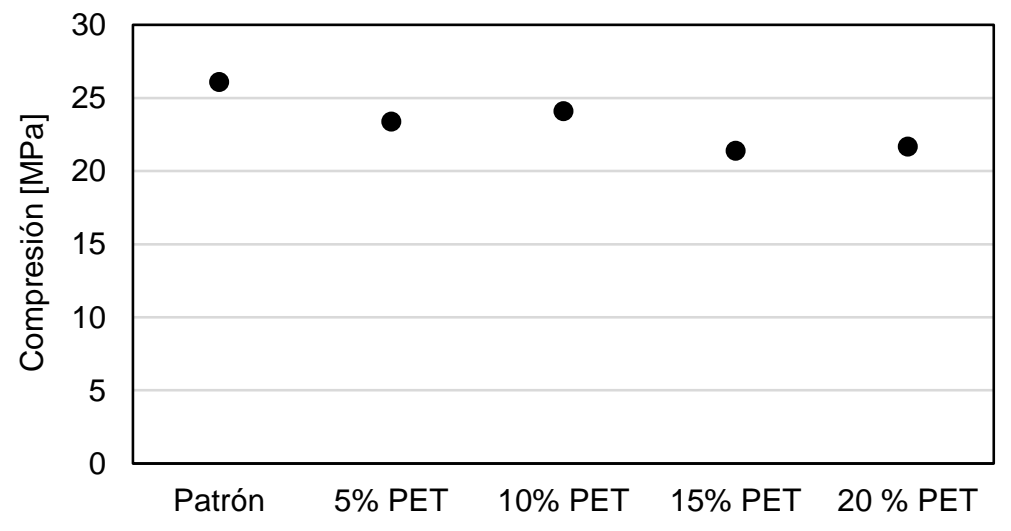

Fig. 3: Resultados ensayos de Compresión

\section{Ensayo absorción de agua}

Con los datos obtenidos se evidencia una proporcionalidad en la disminución de la absorción de agua de modo muy pareja con excepción de la mezcla con $20 \%$ de PET. Se confirma la hipótesis planteada para la gran mayoría de las mezclas. Lo que sucede con la probeta con $20 \%$ PET puede que se deba a que con mayor cantidad de PET existe un punto en que la mezcla deja de ser homogénea y las partes de plástico no se adhieren de buena manera provocando aumento en la porosidad del material. 


\section{Ensayo penetración de cloruros por capilaridad}

Los resultados se muestran disparejos para el ensayo de 48 horas ya que para cada ciclo se utilizaron probetas diferentes con distinta masa, pero a modo general se logra apreciar que a medida que aumenta la cantidad de PET, la variación porcentual en masa disminuye. A las 48 horas del ensayo las probetas que contienen $10 \%$ de PET son las menos afectadas. Y las de mayores penetraciones son las de $15 \%$ PET (ver figura $\left.{ }^{\circ} 4\right)$. Los resultados obtenidos no dilucidan el comportamiento esperado en las probetas con adición de PET, que debiera ser similar a los resultados obtenidos en el ensayo de absorción de agua. A pesar de la conclusión de este ensayo no es directa, se cree que la razón se debe a filtraciones por las caras exteriores de las probetas donde se forma la lechada, por tener menor cantidad de áridos le es más fácil a la solución de cloruros penetrar por los costados.

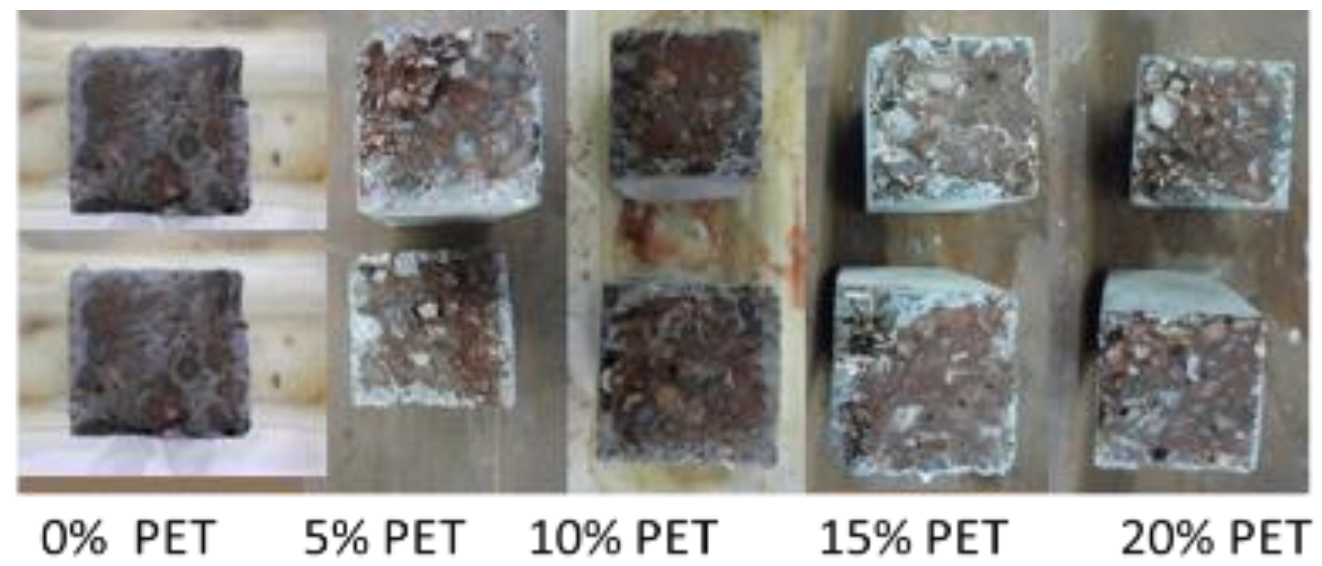

Fig. 4: Resultados de la penetración de cloruros por capilaridad a las 48 horas

\section{Resultados económicos}

A continuación se presentan los costos que genera la fabricación de este material por volumen para cada dosificación estudiada. En la tabla 4 se visualiza los costos (en pesos chilenos) de fabricación de cada una de las mezclas estudiadas

Tabla 4: Costos por $\mathrm{m}^{3}$ de producto

\begin{tabular}{|c|c|c|c|c|c|c|}
\hline Materia Prima & $\begin{array}{l}\text { Precio } \\
\text { Unitario } \\
(\$ C L P)\end{array}$ & Patrón & $\begin{array}{c}\text { Mezcla 5\% } \\
\text { Pet }\end{array}$ & $\begin{array}{c}\text { Mezcla } 10 \% \\
\text { Pet }\end{array}$ & $\begin{array}{c}\text { Mezcla } 15 \% \\
\text { Pet }\end{array}$ & $\begin{array}{c}\text { Mezcla } 20 \% \\
\text { Pet }\end{array}$ \\
\hline PET (kg) & 200 & 0 & 2.400 & 5.000 & 7.400 & 9.800 \\
\hline Cemento $(\mathrm{kg})$ & 4.550 & 39.933 & 39.933 & 39.933 & 39.933 & 39.933 \\
\hline Arena $\left(\mathrm{m}^{3}\right)$ & 14.100 & 9.038 & 8.587 & 8.136 & 7.685 & 7.233 \\
\hline Gravilla $\left(\mathrm{m}^{3}\right)$ & 11.200 & 6.082 & 6.082 & 6.082 & 6.082 & 6.082 \\
\hline Total $\left(\$ / \mathrm{m}^{3}\right)$ & & 55.053 & 57.002 & 59.151 & 61.100 & 63.048 \\
\hline
\end{tabular}

En la tabla 4 se se observa el aumento progresivo del PET incide de la misma manera en el precio final, debido a que se estudió la compra de PET directa a una empresa recicladora, se recomienda estudiar los costos para fabricar el PET de manera autónoma e industrializada. El ahorro de arena es desde $\$ 451$ por $\mathrm{m}^{3}$ en el caso de reemplazar el $5 \%$ hasta $\$ 1805$ por $\mathrm{m}^{3}$ para el material con un reemplazo de $20 \%$ de PET, a fin de cuenta se reemplaza el agregado más costoso por un material reciclado. Ya conocido el precio por $\mathrm{m}^{3}$ de material, se procede a ser el cálculo unitario del producto final, con el objeto de comparar con los otros productos del mercado para la construcción de albañilería. Las medidas son respaldadas para la fabricación de ladrillos de hormigón silíceo NCh180 Of57. En el caso de los bloques se utilizaron medidas comerciales de bloques marca GRAU. En la figura 5 se presentan lo precios comerciales de diferentes productos de albañilería, tanto de ladrillos de arcilla como de bloques de hormigón. 


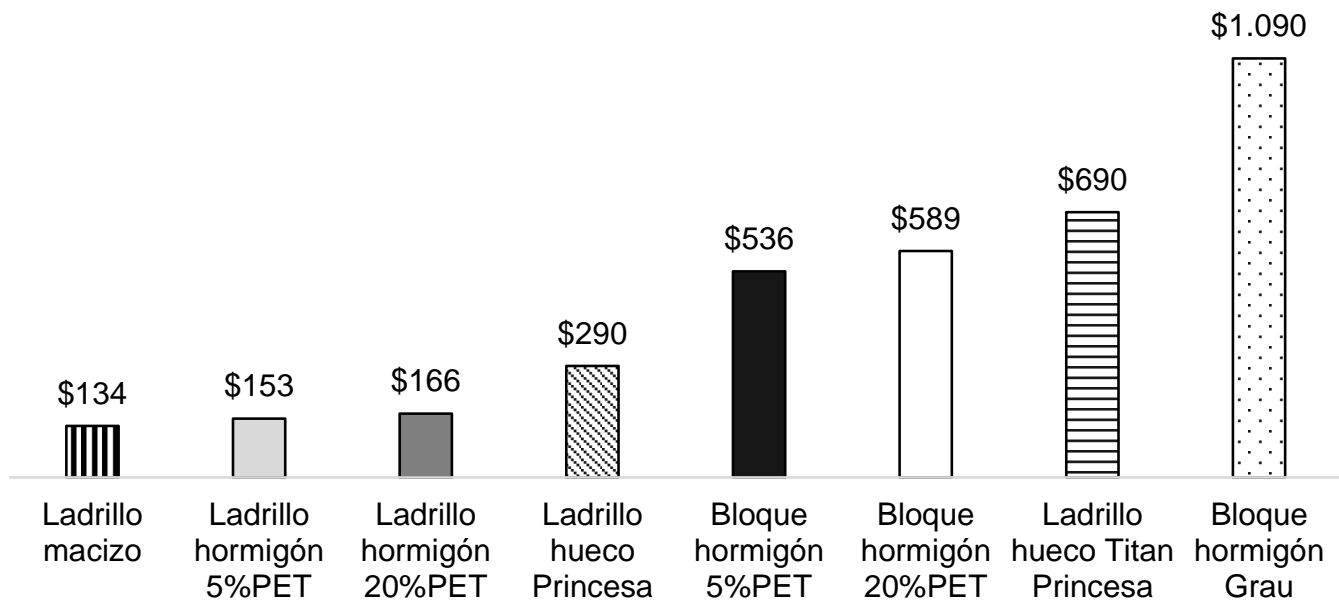

Fig. 5: Comparativo entre los competidores de bloques con PET

Es altamente competitivo el precio calculado para el bloque de hormigón con incorporación de PET, el que fluctúa de $\$ 536$ a $\$ 589$, además de tratarse de un producto más eficiente en la albañilería por su rapidez de montaje y su volumen es que los bloques existentes en el mercado duplican ( $\$ 1090$ para el competidor de Grau) el precio de un bloque de hormigón con agregado de PET.Para el caso de los ladrillos el mercado es más variable, se observan valores desde los $\$ 134$ para el ladrillo macizo hasta los $\$ 690$ en el caso de ladrillo hueco Titán, ya que ofrecen productos de arcilla con diferentes características con la finalidad de mejorar sus propiedades, las cuales en el ladrillo de hormigón vienen inherente sin la necesidad de agregar aditivos al material. El costo calculado es para un ladrillo macizo de hormigón que compite altamente también con todos los materiales ofrecidos, debido que el rango va desde los \$153 a \$166 la unidad.

\section{Resultados medioambientales}

Los cálculos de consumo energético y emisión de $\mathrm{CO} 2$ en las etapas de extracción de materias primas, transporte y fabricación de los ladrillos de hormigón se presentan en la tabla 5. Se obtiene que en las etapas calculadas, de extracción y procesamiento de materias primas, transporte y fabricación del ladrillo de hormigón con PET reciclado emite $16 \mathrm{kgCO}_{2 \text { eq. }}$.

Tabla 5: Emisión de $\mathrm{CO}_{2}$ eq y consumo energético para todo el ciclo de vida del producto

\begin{tabular}{|l|l|r|r|}
\hline \multicolumn{2}{|c|}{ Etapas } & $\mathrm{KgCO}_{2}$ & $\mathrm{Kwh} / \mathrm{M}^{3}$ \\
\hline \multirow{4}{*}{$\begin{array}{l}\text { Extracción } \\
\text { Materias Primas }\end{array}$} & Agregado Grueso & 0,30 & 7,65 \\
\cline { 2 - 4 } & Agregado Fino & 0,67 & 24,58 \\
\cline { 2 - 4 } & Cemento & 14,64 & 484,58 \\
\cline { 2 - 4 } & PET (10\%) & 0,27 & 6,50 \\
\cline { 2 - 4 } & TOTAL & 15,88 & 523,31 \\
\hline Transporte & 2,62 & 87,2 \\
\hline Fabricación & 0,0004 & 0,96 \\
\hline Construcción & 1,98 & 65,41 \\
\hline Uso & 11,24 & 370,6 \\
\hline Demolición & 1,32 & 43,6 \\
\hline
\end{tabular}

Como se puede visualizar en la tabla 5 las materias primas poseen un importante consumo energético en el ciclo del producto, siendo considerable la emisión de $\mathrm{CO}_{2}$ que genera. El proceso de fabricación casi no incide ya que consume $0,96 \mathrm{kWh} / \mathrm{m}^{3}$ y emiten sólo $0,0004 \mathrm{kgCO}$ por $\mathrm{m}^{3}$ por las maquinarias utilizadas. En la figura 6 se evidencia el alto impacto que tiene el cemento en el medio ambiente, además tiene una incidencia del $92 \%$ de la emisión de $\mathrm{CO}_{2 \text { eq }}$ en la extracción y procesamiento de materias primas...Cabe destacar que el PET está siendo reemplazado por el segundo agregado más contaminante, la arena, además disminuye el problema de extracción de áridos (Valdés \& Rapiman, 2007) y la emisión de $\mathrm{CO}_{2}$ al ambiente. 


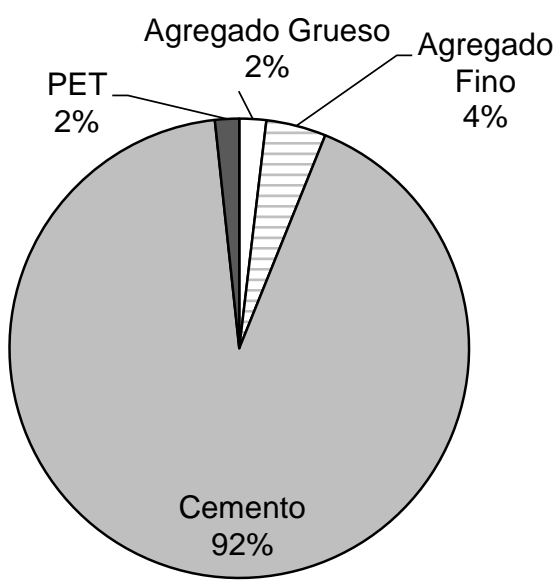

Fig. 6: Incidencia de las Materias Primas en las emisiones de $\mathrm{CO}_{2}$ para la etapa de extracción y procesamiento

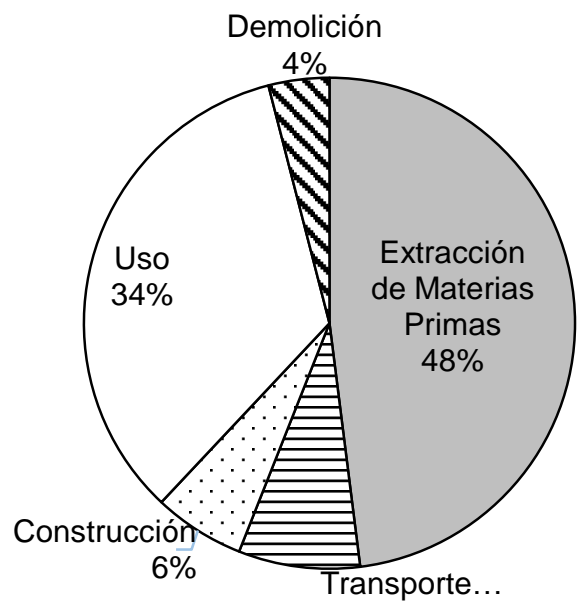

Fig. 7: Distribución de las emisiones de $\mathrm{CO}_{2}$ en las distintas etapas del ciclo de vida

Con los supuestos recogidos se observa en la figura 7 que la etapa más contaminante es la de la extracción de materias primas, esto se debe al uso del cemento en el material, tal como se vio este impacta enormemente en el cálculo de la huella de carbono. El proceso de fabricación no se visualiza ya que consume $0,96 \mathrm{kWh} / \mathrm{m}^{3}$ por las maquinarias utilizadas. Del mismo modo al tratarse de maquinarias eléctricas estas emiten 0,0004 $\mathrm{kgCO}_{2}$ por $\mathrm{m}^{3}$ de material. La etapa de Uso y mantención también emite grandes cantidades de $\mathrm{CO}_{2}$, esto es debido a la calefacción y refrigeración del hogar durante la vida de la vivienda.En así como en este tipo de material es importante buscar alternativas o procesos más eficientes en la producción del cemento. De todos modos se debe procurar hacer todos los procesos eficientes con los menores consumos energéticos y controlados, para reducir al mínimo las emisiones de $\mathrm{CO}_{2}$ generadas.

\section{DISCUSIÓN FINAL}

De acuerdo a los resultados obtenidos, en el aspecto económico, en la utilización de bloques con agregado PET se abaratan los costos de construcción ya que no requiere de mano de obra calificada para su implementación, incluso puede ser autoconstruido por las familias que vivirán en ella. Los materiales a emplear también resultan más económicos por tratarse de residuos y no requiere de su extracción y procesamiento. Por lo tanto es un material altamente competitivo en el mercado ya que los ladrillos con adición de PET logran ser la mitad más económicos que un ladrillo convencional. Técnicamente resulta ser un material adecuado para su utilización en albañilería, con una resistencia mecánica a compresión mayor a $20 \mathrm{MPa}$ cumpliendo con las normas chilenas de ladrillos que exige 7MPa. También lo hacen levemente más liviano, ya que el aumentar el porcentaje de reemplazo de PET disminuye la densidad, contribuyendo un menor peso de la estructura. Y posee un mejor comportamiento frente a la absorción de agua a medida que aumenta la cantidad de PET. 
Ambientalmente luego de su cálculo de huella de carbono se presume que es un material más sustentable que el hormigón convencional y que los ladrillos cerámicos, pero continúa siendo altamente contaminante por contener cemento (92\% de la emisión de $\mathrm{CO}_{2 \text { eq }}$ en la extracción y procesamiento). El material con un reemplazo de arena de $10 \%$ de $\mathrm{PET}$ reciclado emite $33,08 \mathrm{kgCO}_{2 \text { eq }}$ por $\mathrm{m}^{3}$ de material en todo su ciclo de vida.

\section{CONCLUSIONES}

La solución propuesta aporta a la reducción del uso de recursos naturales en los materiales de construcción, reciclando uno de los residuos de mayor consumo en Chile, el plástico, que impacta significativamente al medio ambiente.

Al concientizar a la sociedad de que los residuos pueden ser reutilizados o reciclados para otros materiales, la cifra de reciclaje de un $4 \%$ de las 668.000 toneladas de plástico generadas en Chile aumentará, aprovechándose las medidas gubernamentales y los intereses empresariales por aprovechar estos materiales.

De acuerdo a los resultados obtenidos, se abren oportunidades de desarrollo para investigar más sobre los materiales de construcción sustentables y sobre las posibilidades de reducir los residuos industriales, domiciliarios y de la industria de la construcción, ya que con esta investigación se ha demostrado que este nuevo material es competitivo económica y medioambientalmente.

\section{REFERENCIAS}

Alesmar, L., N. Rendón y M. Korody, Diseños de mezcla de tereftalato de polietileno (PET)-cemento, Revista de la Facultad de Ingeniería Universidad Central de Venezuela, 23(1), 76-86 (2008)

Athena Sustainable Materials Institute, Life cycle analysis of bricks and mortar products, $1^{\text {a }}$ Ed., $1-114$, George J. Venta P. Eng, Otawa, Canada (1998)

C y V Medioambiente, Diagnóstico producción, importación y distribución de envases y embalajes y el manejo de los residuos de envases y embalajes, 1-185, Ministerio del Medio Ambiente - Gobierno de Chile, Santiago-Chile (2010)

Choi, Y. W., D. J. Moon, J. S. Chung y S. K. Cho, Effects of waste PET bottles aggregate on the properties of concrete, doi: 10.1016/j.cemconres.2004.05.014, Cement and concrete research, 35(4), 776-781 (2005)

Comisión Nacional del Medio Ambiente. Primer reporte sobre manejo de residuos sólidos en Chile. 1-64, Departamento de Comunicaciones CONAMA, Santiago-Chile (2010)

Costa Del Pozo, A., Estudio de hormigones y morteros aligerados con agregados de plástico reciclado como árido y carga en la mezcla, Tesis de Magíster, Escuela de Arquitectura, Energía y Medio Ambiente Universidad Politécnica de Cataluña, Barcelona-España (2012)

Gaggino, R., R. Arguello y H. Berretta, Aplicación de material plástico reciclado en elementos constructivos a base de cemento, IV Encuentro Nacional y II Latinoamericano, Edificaciones y Comunidades sustentables, 906-915, Campo Grande, Brasil (2007)

García de los Santos, E., M. Martínez-Mateo y otros 7 autores, Estudio de factibilidad bloques de hormigón con agregado de residuo plástico ABS, 11th LACCEI Latin American and Caribbean Conference for Engineering and Technology, 1-10, Cancún-México, 14-16 Agosto (2013)

Gómez, M., A.M. Carvajal y V. Santelices, Influencia del Polietileno de Alta Densidad (PEAD) usado como adición en el mortero de Cemento, doi: 10.4067/S0718-915X2011000300011, Revista de la Construcción, 10(3), 110-121 (2011)

Guo, S., J. Hu y Q. Dai, A critical review on the performance of Portland cement concrete with recycled organic components, doi: 10.1016/j.jclepro.2018.03.244, Journal of Cleaner Production, 188, 92-112 (2018)

Gupta, T., S. Chaudhary y R.K. Sharma, Assessment of mechanical and durability properties of concrete containing waste rubber tire as fine aggregate, doi: 10.1016/j.conbuildmat.2014.09.102, Construction and Building Materials, 73, 562-574 (2014)

ISO 14040:2006(es): Organización Internacional de Normalización (Suiza). Gestión ambiental - Análisis del ciclo de vida - Principios y marco de referencia, 1-20, Ginebra-Suiza (2006)

Kim, S., N. Yi, H. Kim, J. Kim e Y. Song, Material and structural performance evaluation of recycled PET fiber reinforced concrete, doi: 10.1016/j.cemconcomp.2009.11.002, Cement and Concrete Composites, 30, 112-121 (2009)

Martínez-Barrera, G., M. Martínez-López y E. Martínez-Cruz, Concreto polimérico reforzado con fibras de luffa, Información Tecnológica, 24(4), 67-74 (2013)

Mendivil-Escalante, J. M., J. M. Gómez-Soberón, J. L. Almaral-Sánchez y F. G. Cabrera-Covarrubias, Metamorphosis in the Porosity of Recycled Concretes Through the Use of a Recycled Polyethylene Terephthalate (PET) Additive, Correlations between the Porous Network and Concrete Properties, doi: 10.3390/ma10020176, Materials, 10(2), 176 (2017) 
NCh1116: Instituto Nacional de Normalización (Chile). Áridos para morteros y hormigones - Determinación de la densidad aparente, 1-10, Santiago-Chile (2008)

NCh1117: Instituto Nacional de Normalización (Chile). Áridos para morteros y hormigones - Determinación de las densidades real y neta y la absorción de agua de las gravas, 1-10, Santiago-Chile (2010)

NCh1223: Instituto Nacional de Normalización (Chile). Áridos para morteros y hormigones - Determinación del material fino menor a 0,080 mm, 1-7, Santiago-Chile (1977)

NCh1239: Instituto Nacional de Normalización (Chile). Áridos para morteros y hormigones - Determinación de las densidades reales y neta de la absorción de agua de las arenas, 1-11, Santiago-Chile (2009)

NCh1326: Instituto Nacional de Normalización (Chile) Áridos para morteros y hormigones: - Determinación de huecos, 1 7, Santiago-Chile (2012)

NCh158: Instituto Nacional de Normalización (Chile). Cementos - Ensayo de flexión y compresión de morteros de cemento, 1-13, Santiago-Chile (1967)

NCh165: Instituto Nacional de Normalización (Chile). Áridos para morteros y hormigones - Tamizado y determinación de la granulometría, 1-17, Santiago-Chile (2009)

Nikbin, I. M., S. Rahimi, H. Allahyari y F. Fallah, Feasibility study of waste Poly Ethylene Terephthalate (PET) particles as aggregate replacement for acid erosion of sustainable structural normal and lightweight concrete, Journal of Cleaner Production, doi: 10.1016/j.jclepro.2016.02.143, 126, 108-117 (2016)

Orasutthikul, S., D. Unno y H. Yokota, Effectiveness of recycled nylon fiber from waste fishing net with respect to fiber reinforced mortar, doi: 10.1016/j.conbuildmat.2017.04.134, Construction and Building Materials, 146, 594-602 (2017)

Pacheco-Torgal, F., Y. Ding y S. Jalali, Properties and durability of concrete containing polymeric wastes (tyre rubber and polyethylene terephthalate bottles): An overview, doi:10.1016/j.conbuildmat.2011.11.047, Construction and Building Materials, 30, 714-724 (2012)

Saikia, N. y J. de Brito, Mechanical properties and abrasion behaviour of concrete containing shredded PET bottle waste as a partial substitution of natural aggregate, doi: 10.1016/j.conbuildmat.2013.11.049, Construction and building materials, 52, 236-244 (2014)

Tafheem, Z., R.I. Rakib y otros tres autores, Experimental investigation on the properties of concrete containing postconsumer plastic waste as coarse aggregate replacement, Journal of Materials and Engineering Structures, 5(1), 23-31 (2018)

Valdés, G.A. y J.G. Rapimán, Propiedades físicas y mecánicas de bloques de hormigón compuestos con áridos reciclados, Información Tecnológica, 18(3), 81-88 (2007)

Vélez-Herrera, J., S. Velásquez-Restrepo, D. Giraldo-Vásquez y P. Castaño-Rivera, Aplicación de microesferas poliméricas expandibles en la elaboración de materiales: Una revisión de la literatura, Informador Técnico, 80(2), $159-168$ (2016)

Yang, S., X. Yue, X. Liu e Y. Tong, Properties of self-compacting lightweight concrete containing recycled plastic particles, doi: 10.1016/j.conbuildmat.2015.03.038, Construction and Building Materials, 84, 444-453 (2015) 
